\title{
NOTE ON GREEN'S THEOREM.
}

BY MR. C. A. EPPERSON.

(Read before the American Mathematical Society April 24, 1915.)

1. Introduction.-We wish in this paper to extend Green's theorem to apply to relations equivalent to the general linear partial differential equation of the second order in two variables. The relations are written in such a way as not to involve derivatives of the second order, and the theorem is proved without assuming their existence.* This point of view is desirable for the possibility of its application to physics.

2. Statement of Lemma.-We shall denote by $a_{i j}, a_{i}, a$, arbitrary continuous functions of $x$ and $y$, the $a_{i j}$ to have also continuous first and second partial derivatives, and the $a_{i}$ to have continuous first partial derivatives, over a given simply connected region. By $b_{i j}, b_{i}, b$, we indicate the coefficients of the linear partial differential expression of the second order which is adjoint to the expression of which the coefficients are $a_{i j}, a_{i}$, $a$. That is, $b_{i j}=a_{i j}$,

$$
\begin{gathered}
b_{i}=2 \frac{\partial a_{i 1}}{\partial x}+2 \frac{\partial a_{i 2}}{\partial y}-a_{i}, \\
b=\frac{\partial^{2} a_{11}}{\partial x^{2}}+\frac{\partial^{2} a_{22}}{\partial y^{2}}+2 \frac{\partial^{2} a_{12}}{\partial x \partial y}-\frac{\partial a_{1}}{\partial x}-\frac{\partial a_{2}}{\partial y}+a .
\end{gathered}
$$

* The immediate occasion for the publication of this paper was the appearance of an article by C. W. Oseen, "Über einen Satz von Green und über die Definitionen von Rot und Div," Rendiconti del Circolo Matematico di Palermo, vol. 38 (1914), pp. 167-179, which applies this method, by means of the principles of vector analysis, to the special case of Poisson's equation. The general proof is a first step in the detailed working out of a general problem for partial differential equations (see preliminary communication of December, 1913, G. C. Evans, "Green's functions for linear partial differential expressions of the second order, and Green's theorem," Bulletin, vol. 20, no. 6, March, 1914).

A proof has been given for equations of parabolic type, which may be generalized to the equations here considered. (G. C. Evans, "On the reduction of integro-differential equations," Transactions Amer. Math. Society, vol. 15 , no. 4, p. 486, Oct., 1914. This paper also gives the literature of the problem). The proof referred to however, by a reduction, makes use of Green's theorem in its usual form; in the present paper, the result is reduced ab initio. 
The $b_{i j}$ are then continuous with their first and second partial derivatives, the $b_{i}$ continuous with their first partial derivatives, and $b$ continuous over the given region. We shall consider a square $S_{r}$, and by $\lim _{S_{r} \dot{ }}$ we shall mean that no matter how small a circle we take about $p$ the square shall ultimately become and remain entirely within that circle. We have then

Lemma I.* Let $u(x, y)$ and $v(x, y)$ and their first partial derivatives be continuous over the given region in the (xy) plane, and let $p$ be a point within that region. If

$$
\begin{aligned}
(f-b u)_{p}=\lim _{S_{r} \doteq p} \frac{1}{\sigma_{r}} \int_{S_{r}} & {\left[\frac{\partial}{\partial x}\left(b_{11} u\right)+\frac{\partial}{\partial y}\left(b_{12} u\right)-b_{1} u\right] d y } \\
- & {\left[\frac{\partial}{\partial x}\left(b_{12} u\right)+\frac{\partial}{\partial y}\left(b_{22} u\right)-b_{2} u\right] d x }
\end{aligned}
$$

and

$$
\begin{aligned}
(g-a v)_{p}=\lim _{S_{r} \doteq p} \frac{1}{\sigma_{r}} \int_{S_{r}} & {\left[\frac{\partial}{\partial x}\left(a_{11} v\right)+\frac{\partial}{\partial y}\left(a_{12} v\right)-a_{1} v\right] d y } \\
- & {\left[\frac{\partial}{\partial x}\left(a_{12} v\right)+\frac{\partial}{\partial y}\left(a_{22} v\right)-a_{2} v\right] d x }
\end{aligned}
$$

then

$$
\begin{aligned}
& (v f-u g)_{p}=\lim _{S_{r} \doteq p} \frac{1}{\sigma_{r}} \int_{S_{r}}\left[a_{11}\left(v \frac{\partial u}{\partial x}-u \frac{\partial v}{\partial x}\right)\right. \\
& \left.+a_{12}\left(v \frac{\partial u}{\partial y}-u \frac{\partial v}{\partial y}\right)+\frac{a_{1}-b_{1}}{2} u v\right] d y-\left[a_{21}\left(v \frac{\partial u}{\partial x}-u \frac{\partial v}{\partial x}\right)\right. \\
& \left.\quad+a_{22}\left(v \frac{\partial u}{\partial y}-u \frac{\partial v}{\partial y}\right)+\frac{a_{2}-b_{2}}{2} u v\right] d x .
\end{aligned}
$$

3. Proof of Lemma I. - If we multiply equation (1) by $v_{p}$ and equation (2) by $u_{p}$ and subtract, we have, using the relations existing between the $a$ 's and $b$ 's of these equations,

* The reader will note that if the second derivatives of $u$ and $v$ exist the theorem corresponds to setting

$$
f=a_{11} \frac{\partial^{2} u}{\partial x^{2}}+a_{22} \frac{\partial^{2} u}{\partial y^{2}}+2 a_{12} \frac{\partial^{2} u}{\partial x \partial y}+a_{1} \frac{\partial u}{\partial x}+a_{2} \frac{\partial u}{\partial y}+a u
$$

and $g=$ the adjoint of $f$. We then have the ordinary Green's theorem. 


$$
\begin{aligned}
(v f & -u g)_{p}+\frac{1}{2}\left[\frac{\partial}{\partial x}\left(a_{1}-b_{1}\right)+\frac{\partial}{\partial y}\left(a_{2}-b_{2}\right)\right]_{p} u_{p} v_{p} \\
& =\lim _{s_{r} \doteq p} \frac{1}{\sigma_{r}} \int_{S_{r}}\left[a_{11}\left(v_{p} \frac{\partial u}{\partial x}-u_{p} \frac{\partial v}{\partial x}\right)+a_{12}\left(v_{p} \frac{\partial u}{\partial y}-u_{p} \frac{\partial v}{\partial y}\right)\right. \\
& \left.+\frac{a_{1}-b_{1}}{2}\left(v_{p} u-u_{p} v\right)\right] d y \\
& -\left[a_{21}\left(v_{p} \frac{\partial u}{\partial x}-u_{p} \frac{\partial v}{\partial x}\right)+a_{22}\left(v_{p} \frac{\partial u}{\partial y}-u_{p} \frac{\partial v}{\partial y}\right)\right. \\
& \left.+\frac{a_{2}-b_{2}}{2}\left(v_{p} u-u_{p} v\right)\right] d x .
\end{aligned}
$$

If now we consider the difference function $D$ of the righthand side of the equations (3) and (4), we have

$$
\begin{aligned}
\lim _{s_{r} \doteq p} D= & \lim _{s_{r} \dot{ }=p} \frac{1}{\sigma_{r}} \int_{s_{r}}\left\{a_{11}\left[\left(v_{p}-v_{s}\right) \frac{\partial u}{\partial x}-\left(u_{p}-u_{s}\right) \frac{\partial v}{\partial x}\right]\right. \\
& +a_{12}\left[\left(v_{p}-v_{s}\right) \frac{\partial u}{\partial y}-\left(u_{p}-u_{s}\right) \frac{\partial v}{\partial y}\right] \\
& \left.+\frac{a_{1}-b_{1}}{2}\left[\left(v_{p}-v_{s}\right) u_{s}+u_{p} v_{s}\right]\right\} d y \\
& -\left\{a_{21}\left[\left(v_{p}-v_{s}\right) \frac{\partial u}{\partial x}-\left(u_{p}-u_{s}\right) \frac{\partial v}{\partial x}\right]\right. \\
& +a_{22}\left[\left(v_{p}-v_{s}\right) \frac{\partial u}{\partial y}-\left(u_{p}-u_{s}\right) \frac{\partial v}{\partial y}\right] \\
& \left.+\frac{a_{2}-b_{2}}{2}\left[\left(v_{p}-v_{s}\right) u_{s}+u_{p} v_{s}\right]\right\} d x .
\end{aligned}
$$

In order to establish the lemma it is sufficient to show that

$$
\lim _{s_{r} \doteq p} D=\frac{1}{2}\left[\frac{\partial}{\partial x}\left(a_{1}-b_{1}\right)+\frac{\partial}{\partial y}\left(a_{2}-b_{2}\right)\right]_{p} u_{p} v_{p} .
$$

To obtain the value of $D$ we will divide the square into four smaller rectangles by lines through $p$ parallel to the sides of the square and consider the sum of the integrals extended around these smaller rectangles. For convenience of notation 
we shall consider $p$ as the origin, and the vertices of the square lettered as in accompanying figure (Fig. 1). We will write

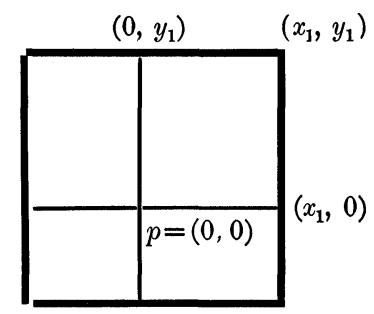

Fig. 1.

the curvilinear integral $D_{1}$ around one of these small rectangles, say $\left[(0,0) /\left(x_{1}, y_{1}\right)\right]$, as a simple integral.

Thus

$$
\begin{aligned}
D_{1}= & \frac{1}{\sigma_{r}} \int_{0}^{y_{1}}\left[\left\{a_{11}\left[\left(v_{p}-v_{s}\right) \frac{\partial u}{\partial x^{-}}-\left(u_{p}-u_{s}\right) \frac{\partial v}{\partial x}\right]\right.\right. \\
& +a_{12}\left[\left(v_{p}-v_{s}\right) \frac{\partial u}{\partial y}-\left(u_{p}-u_{s}\right) \frac{\partial v}{\partial y}\right] \\
& \left.+\frac{a_{1}-b_{1}}{2}\left[\left(v_{p}-v_{s}\right) u_{s}+u_{p} v_{s}\right]\right\}_{x_{1}, y} \\
& -\left\{a_{11}\left[\left(v_{p}-v_{s}\right) \frac{\partial u}{\partial x}-\left(u_{p}-u_{s}\right) \frac{\partial v}{\partial x}\right]\right. \\
& +a_{12}\left[\left(v_{p}-v_{s}\right) \frac{\partial u}{\partial y}-\left(u_{p}-u_{s}\right) \frac{\partial v}{\partial y}\right] \\
& \left.\left.+\frac{a_{1}-b_{1}}{2}\left[\left(v_{p}-v_{s}\right) u_{s}+u_{p} v_{s}\right]\right\}_{0, y}\right] d y
\end{aligned}
$$

plus the corresponding term to be integrated with respect to $x$.

If we note the figure we see that

$$
\begin{aligned}
& \left|\left(v_{s}-v_{p}\right)-\left(\frac{\partial v_{s}}{\partial x} x_{1}+\frac{\partial v_{s}}{\partial y} y\right)\right|<\epsilon\left(\left|x_{1}\right|+|y|\right)<2 \epsilon \delta \\
& 0 \leq y \leq y_{1}, \quad 0 \leq x \leq x_{1}, \quad \delta=\text { one side of the square, }
\end{aligned}
$$

if $v_{s}$ has the coordinates $\left(x_{1}, y\right)$, and a similar expression holds if $v_{s}$ is on one of the other sides. Similar expressions hold 
for $\left(u_{s}-u_{p}\right)$. Further, one infinitesimal $\epsilon$ may be used for all such expressions, as $u$ and $v$ and their first partial derivatives are uniformly continuous throughout the square. In the limit then $\left(v_{s}-v_{p}\right)$ may be replaced by $\left(\partial v_{s} / \partial x\right) x_{1}+\left(\partial v_{s} / \partial y\right) y$ or by the corresponding expressions depending on the position of $v_{s}$.

If we carry out these substitutions in detail we obtain an expression of the form

$$
\begin{aligned}
D_{1}= & \frac{1}{\sigma_{r}} \int_{0}^{y_{1}}\left[\left\{\left[a_{11}\left(\frac{\partial v}{\partial y} \frac{\partial u}{\partial x}-\frac{\partial u}{\partial y} \frac{\partial v}{\partial x}\right)\right]_{x_{1, y}}\right.\right. \\
& \left.-\left[a_{11}\left(\frac{\partial v}{\partial y} \frac{\partial u}{\partial x}-\frac{\partial u}{\partial y} \frac{\partial v}{\partial x}\right)\right]_{0, y}\right\}(-y) \\
& +\left\{\left[a_{12}\left(\frac{\partial v}{\partial x} \frac{\partial u}{\partial y}-\frac{\partial u}{\partial x} \frac{\partial v}{\partial y}\right)\right]_{x_{1}, y}\right. \\
& \left.-\left[a_{12}\left(\frac{\partial v}{\partial x} \frac{\partial u}{\partial y}-\frac{\partial u}{\partial x} \frac{\partial v}{\partial y}\right)\right]_{0, y}\right\}\left(-x_{1}\right) \\
& +\left\{\left[\frac{a_{1}-b_{1}}{2}\left(\frac{\partial v}{\partial y} u\right)\right]_{x_{1}, y}\right. \\
& \left.-\left[\frac{a_{1}-b_{1}}{2}\left(\frac{\partial v}{\partial y} u\right)\right]_{0, y}\right\}(-y) \\
& +u_{p}\left[\left(\frac{a_{1}-b_{1}}{2} v\right)_{x_{1}, y}-\left(\frac{a_{1}-b_{1}}{2} v\right)_{0, y}\right] \\
& +\left(\frac{\left.\left.a_{1}-b_{1} \frac{\partial v}{\partial x} u\right)_{x_{1}, y}\left(-x_{1}\right)\right] d y}{2}\right.
\end{aligned}
$$

plus exactly similar terms to be integrated with respect to $x$, plus terms every one of which is in absolute value less than $\epsilon$ times a constant less than $\left|\sigma_{r}\right|$.

If we apply the law of the mean every term, except the last two, is of one of the forms
(a) $\left[P\left(x_{1}, y\right)-P(0, y)\right]\left(\frac{x_{1} y_{1}}{\sigma_{r}}\right)$ or $\left[P\left(x_{1}, y\right)-P(0, y)\right]\left(\frac{x_{1} x_{1}}{\sigma_{r}}\right)$

$$
\epsilon\left(\frac{x_{1} y_{1}}{\sigma_{r}}\right) \text { or } \epsilon\left(\frac{x_{1} x_{1}}{\sigma_{r}}\right), \text { etc., }
$$


where $P(x, y)$ is uniformly continuous in $x$ and in $y$ throughout the square. Hence in the limits every term except the last two vanishes and we have

$$
D_{1}=\frac{1}{\sigma_{r}}\left[\left(\frac{a_{1}-b_{1}}{2} \frac{\partial v}{\partial x} u\right)_{x_{1}, y}\left(-x_{1}\right)\right.
$$

where $\lim _{S_{r} \doteq p} \eta=0$,

$$
\left.+u_{p} \frac{\partial}{\partial x}\left(\frac{a_{1}-b_{1}}{2} v\right)_{0, y}\left(x_{1}\right)\right] y_{1}+\eta,
$$

$=\frac{1}{\sigma_{r}} u_{p}\left[\left(\frac{a_{1}-b_{1}}{2} \frac{\partial v}{\partial x}\right)_{x_{1}, y}\left(-x_{1}\right)+\left(\frac{a_{1}-b_{1}}{2} \frac{\partial v}{\partial x}\right)_{0, y}\left(x_{1}\right)\right.$

$$
\left.+\left(v \frac{\partial}{\partial x} \frac{a_{1}-b_{1}}{2}\right)_{0, y}\left(x_{1}\right)\right] y_{1}+\eta+\eta^{\prime},
$$

where

$$
\left|\eta^{\prime}\right|<\epsilon\left|\frac{a_{1}-b_{1}}{2} \frac{\partial v}{\partial x}\right|,
$$

which gives us, if we combine with it the corresponding terms from the integration with respect to $x$,

$$
D_{1}=\left(\frac{x_{1} y_{1}}{\sigma_{r}}\right) \frac{1}{2}\left[\frac{\partial}{\partial x}\left(a_{1}-b_{1}\right)+\frac{\partial}{\partial y}\left(a_{2}-b_{2}\right)\right]_{p} u_{p} v_{p}+\omega,
$$

where $\lim _{S_{r} \doteq p} \omega=0, \omega$ being less in absolute value than $\epsilon \delta^{2} / \sigma_{r}$ times some constant.

If we now carry the integration around the other rectangles we have similar expressions for $D_{2}, D_{3}$, and $D_{4}$.

Since $\left(x_{1} y_{1}+x_{2} y_{1}+x_{2} y_{2}+x_{1} y_{2}\right) / \sigma_{r}=1$, we have

$$
\lim _{S_{r}=p} D=\frac{1}{2}\left[\frac{\partial}{\partial x}\left(a_{1}-b_{1}\right)+\frac{\partial}{\partial y}\left(a_{2}-b_{2}\right)\right]_{p} u_{p} v_{p} .
$$

The lemma is therefore established.

4. Statement of Green's Theorem.-By a standard curve we shall mean a closed regular curve which does not cut itself and which cannot be cut in more than a finite number of points by any horizontal or vertical straight line.

We may now state Green's theorem.

THEOREM I. If $u(x, y)$ and $v(x, y)$ with their first partial derivatives, and $f$ and $g$, are continuous throughout a simply 
connected region, and if for every standard curve $S,{ }^{*}$ contained wholly within that region,

$$
\begin{aligned}
\iint_{\sigma}(f-b u) d x d y=\int_{S} & {\left[\frac{\partial}{\partial x}\left(b_{11} u\right)+\frac{\partial}{\partial y}\left(b_{12} u\right)-b_{1} u\right] d y } \\
& -\left[\frac{\partial}{\partial x}\left(b_{21} u\right)+\frac{\partial}{\partial y}\left(b_{22} u\right)-b_{2} u\right] d x
\end{aligned}
$$

and

then

$$
\begin{aligned}
\iint_{\sigma}(g-a v) d x d y=\int_{S} & {\left.\left[\frac{\partial}{\partial x}\left(a_{11} v\right)+\frac{\partial}{\partial y}\left(a_{12} v\right)-a_{1} v\right)\right] d y } \\
& -\left[\frac{\partial}{\partial x}\left(a_{21} v\right)+\frac{\partial}{\partial y}\left(a_{22} v\right)-a_{2} v\right] d x
\end{aligned}
$$

$$
\begin{aligned}
\iint_{\sigma}(v f- & u g) d x d y=\int_{S}\left[a_{11}\left(v \frac{\partial u}{\partial x}-u \frac{\partial v}{\partial x}\right)\right. \\
& \left.+a_{12}\left(v \frac{\partial u}{\partial y}-u \frac{\partial v}{\partial y}\right)+\frac{a_{1}-b_{1}}{2} u v\right] d y \\
& -\left[a_{21}\left(v \frac{\partial u}{\partial x}-u \frac{\partial v}{\partial x}\right)+a_{22}\left(v \frac{\partial u}{\partial y}-u \frac{\partial v}{\partial y}\right)\right. \\
& \left.+\frac{a_{2}-b_{2}}{2} u v\right] d x
\end{aligned}
$$

Proof of Theorem I.-For convenience in writing our equations we will set

$$
A_{i}=a_{i 1}\left(v \frac{\partial u}{\partial x}-u \frac{\partial v}{\partial x}\right)+a_{i 2}\left(v \frac{\partial u}{\partial y}-u \frac{\partial v}{\partial y}\right)+\frac{a_{i}-b_{i}}{2} u v .
$$

Now divide the region enclosed by the contour into a number of squares $\sigma_{i}$ of side $\delta$. Then

$$
\iint_{\sigma}(v f-u g) d x d y=\sum \iint_{\sigma_{i}}(v f-u g) d x_{i} d y_{i}+\eta \quad(n>N),
$$

where $\eta$ is the integral over that portion of $\sigma$ not contained in $\sum^{n} \sigma_{i}$ and can be made as small as we please by choosing $n$ sufficiently large. By the law of the mean

\footnotetext{
* The conclusion holds for the boundary of the region if it is a standard
} curve. 


$$
\iint_{\sigma_{i}}(v f-u g) d x_{i} d y_{i}=(v f-u g)_{p_{i}} \sigma_{i}
$$

where $p_{i}$ is some point in $\sigma_{i}$. Then

$$
\iint_{\sigma}(v f-u g) d x d y=\sum^{n}(v f-u g)_{p_{i}} \sigma_{i}+\eta \quad(n>N) .
$$

The hypotheses of this theorem imply the hypotheses of Lemma I. Hence, by that lemma,

so that

$$
(v f-u g)_{p_{i}}=\lim _{S_{i} \doteq p_{i}} \frac{1}{\sigma_{i}} \int_{S_{i}} A_{1} d y-A_{2} d x,
$$

$$
\begin{aligned}
\iint_{\sigma}(v f-u g) d x d y=\sum^{n}(v f-u g)_{p_{i}} \sigma_{i}+\eta \\
=\sum^{n} \sigma_{i} \lim _{S_{i}=p_{i}} \frac{1}{\sigma_{i}} \int_{S_{i}} A_{1} d y-A_{2} d x .
\end{aligned}
$$

We have to do with a uniform limit in the right-hand member of the above equation, since on account of the assumed continuity of $u, \partial u / \partial x, \partial u / \partial y$ the quantity $\epsilon$ which occurs in the proof of Lemma I depends merely on the size of the square, and not on the position of the point $p$. Hence

differs from

$$
\lim _{S_{i}=p_{i}} \frac{1}{\sigma_{i}} \int_{S_{i}} A_{1} d y-A_{2} d x
$$

$$
\frac{1}{\sigma_{i}} \int_{S_{i}} A_{1} d y-A_{2} d x
$$

by less than $\bar{\epsilon}$, where $\lim _{\delta \doteq 0} \bar{\epsilon}=0$, and

differs from

$$
\sum^{n} \sigma_{i} \lim _{S_{i} \doteq p_{i}} \frac{1}{\sigma_{i}} \int_{S_{i}} A_{i} d y-A_{2} d x
$$

$$
\int_{S_{r}} A_{1} d y-A_{2} d x
$$

by less than $\bar{\epsilon} \sigma$, where $S_{r}$ is the contour formed by the exterior boundary of the approximating squares. It then follows that

$$
\iint_{\sigma}(v f-u g) d x d y=\int_{S_{r}} A_{1} d y-A_{2} d x+\eta^{\prime},
$$


where $\lim _{\delta=0} \eta^{\prime}=0$. But since $A_{1}$ and $A_{2}$ are uniformly continuous in $x$ and $y$ throughout the region, we have

$$
\lim _{\delta \doteq 0} \int_{S_{r}} A_{1} d y-A_{2} d x+\eta^{\prime}=\int_{S} A_{1} d y-A_{2} d x,
$$

and therefore finally

$$
\iint_{\sigma}(v f-u g) d x d y=\int_{S} A_{1} d y-A_{2} d x,
$$

which was to be proved.

Corollary I. Theorem for Poisson's Equation. If $u$ and $v$ and their first partial derivatives, and $f$ and $g$, are continuous throughout a simply connected region, and if for every standard curve $S$ contained wholly within that region,

and

$$
\iint_{\sigma} f(x, y) d x d y=\int_{S} \frac{\partial u}{\partial n} d s
$$

then

$$
\iint_{\sigma} g(x, y) d x d y=\int_{S} \frac{\partial v}{\partial n} d s
$$

$$
\iint_{\sigma}(v f-u g) d x d y=\int_{S}\left(v \frac{\partial u}{\partial n}-u \frac{\partial v}{\partial n}\right) d s .
$$

If we put in this $v=1 / r$ we get the well-known formula due to Green.

Corollary II. By $\lim _{S=p}$ we shall mean that the standard curve $S$ app roaches $p$ uniformly, i. e., if we take a circle with center at $p$ and of arbitrarily small radius the standard curve $S$ ultimately becomes and remains within the circle. We may now state

CoROLLARY II. If $u$ and $v$ and their first partial derivatives are continuous within and on the boundary of a region enclosed by a standard curve $S$ in the (xy) plane, and if

$$
\begin{aligned}
(f-b u)_{p}=\lim _{S \doteq p} \frac{1}{\sigma} \int_{S}\left[\frac{\partial}{\partial x}\left(b_{11} u\right)+\frac{\partial}{\partial y}\left(b_{22} u\right)-b_{2} u\right] d y \\
-\left[\frac{\partial}{\partial x}\left(b_{12} u\right)+\frac{\partial}{\partial y}\left(b_{22} u\right)-b_{2} u\right] d x
\end{aligned}
$$

and 


$$
\begin{aligned}
(g-a v)_{p}=\lim _{S \dot{=}} \frac{1}{\sigma} \int_{S}\left[\frac{\partial}{\partial x}\left(a_{11} v\right)+\frac{\partial}{\partial y}\left(a_{12} v\right)-a_{1} v\right] d y \\
-\left[\frac{\partial}{\partial x}\left(a_{21} v\right)+\frac{\partial}{\partial y}\left(a_{22} v\right)-a_{2} v\right] d x
\end{aligned}
$$

then

$$
\begin{aligned}
(v f-u g)_{p}= & \lim _{S \doteq p} \frac{1}{\sigma} \int_{S}\left[a_{11}\left(v \frac{\partial u}{\partial x}-u \frac{\partial v}{\partial x}\right)+a_{12}\left(v \frac{\partial u}{\partial y}-u \frac{\partial v}{\partial y}\right)\right. \\
& \left.+\frac{a_{1}-b_{1}}{2} u v\right] d y-\left[a_{21}\left(v \frac{\partial u}{\partial x}-u \frac{\partial v}{\partial x}\right)\right. \\
& \left.+a_{22}\left(v \frac{\partial u}{\partial y}-u \frac{\partial v}{\partial y}\right)+\frac{a_{2}-b_{2}}{2} u v\right] d x,
\end{aligned}
$$

where $p$ is any point within $S$.

The Rice Institute, February, 1915.

\title{
CONVERGENCE OF THE SERIES $\sum_{i=0}^{\infty} \sum_{j=0}^{\infty} \frac{x^{i} y^{j}}{i-j \gamma}$ \\ $(\gamma$ IRRATIONAL).
}

\author{
BY PROFESSOR W. D. MACMILLAN.
}

(Read before the American Mathematical Society April 2, 1915.)

The method of proof which is here used depends upon the properties of continued fractions. Any irrational number $\gamma$ can be expanded as a simple continued fraction

$$
\gamma=a_{1}+\frac{1}{a_{2}+}+\frac{1}{a_{3}+}+\frac{1}{a_{4}+} \cdots .
$$

Let $p_{n} / q_{n}$ be the $n$th principal convergent,* and $P / Q$ be any intermediate convergent lying between $p_{n-2} / q_{n-2}$ and $p_{n} / q_{n}$. Then

$$
\frac{p_{n-2}}{q_{n-2}}<\frac{P}{Q}<\frac{p_{n}}{q_{n}}<\gamma<\frac{p_{n+1}}{q_{n+1}}<\frac{p_{n-1}}{q_{n-1}}
$$

if $n$ is odd, and

* The notation used here agrees with that of Chrystal's Algebra, Vol. II, Chap. XXXII. 